\title{
Take Three Snapshots - A Tool for Fast Freehand Acquisition of 3D Objects
}

\author{
Gabriele Peters and Klaus Häming \\ University of Applied Sciences and Arts, Computer Science, Visual Computing, \\ Emil-Figge-Str. 42, D-44221 Dortmund, Germany \\ \{gabriele.peters, klaus.haeming\} afh-dortmund.de
}

\begin{abstract}
We introduce a tool which allows an untrained user to take three images of an object freehand with a simple consumer camera. From these images a $3 \mathrm{~d}$ model of the visible parts of the object is reconstructed within seconds and visualized realistically. From a research point of view we propose solutions for three weaknesses of the state-of-the-art reconstruction pipeline. These contributions allow for a more robust and a considerably faster reconstruction process than before, which can be used, e.g., to create new types of interfaces or to assist in creating virtual environments.
\end{abstract}

Keywords: Object Acquisition, Object Reconstruction, User Interfaces, Interaction Techniques, Visualisation Techniques, Usability.

\section{Introduction}

There is a growing demand for realistic $3 \mathrm{~d}$ models of real-world objects in a large number of fields of applications. One way to obtain such models is the cumbersome manual creation of $3 \mathrm{~d}$ point clouds and meshes, which is still the prevalent method. Automatic methods require complex technical equipment or highly skilled experts or both. Existing methods for object acquisition such as [1] are far from being userfriendly or accessible to the broad public.

\section{A Solution with an Intuitive Interface}

We present a prototype of an intuitive acquisition tool which allows an untrained user to take a few images of an object freehand from slightly different but random viewpoints with an uncalibrated consumer camera. From these images a $3 \mathrm{~d}$ model of the visible parts of the object is reconstructed. The model is visualized as a realistic, freely rotatable model. The scientific background of our demonstration is visualized in Fig. 1. Our contribution to the image processing step consists in a new combination of feature detection and description based on [2] providing a higher robustness. For consistency filtration we have developed a two-stage process based on [3], which facilitates a faster selection of relevant features. Our third contribution consists in a new method to texture-map the point cloud, resulting in an arbitrarily rotatable $3 \mathrm{~d}$ surface. 


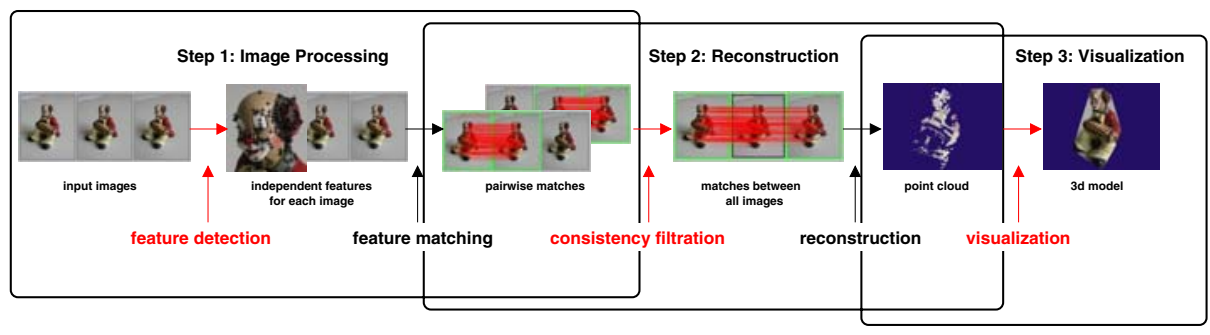

Fig. 1. 1. Image processing with feature detection and matching between image pairs. 2. Filtration of consistent feature matches and reconstruction of $3 \mathrm{~d}$ points. 3 . Visualization with texture mapped on the point cloud. Those parts of this pipeline we contribute to are highlighted red.
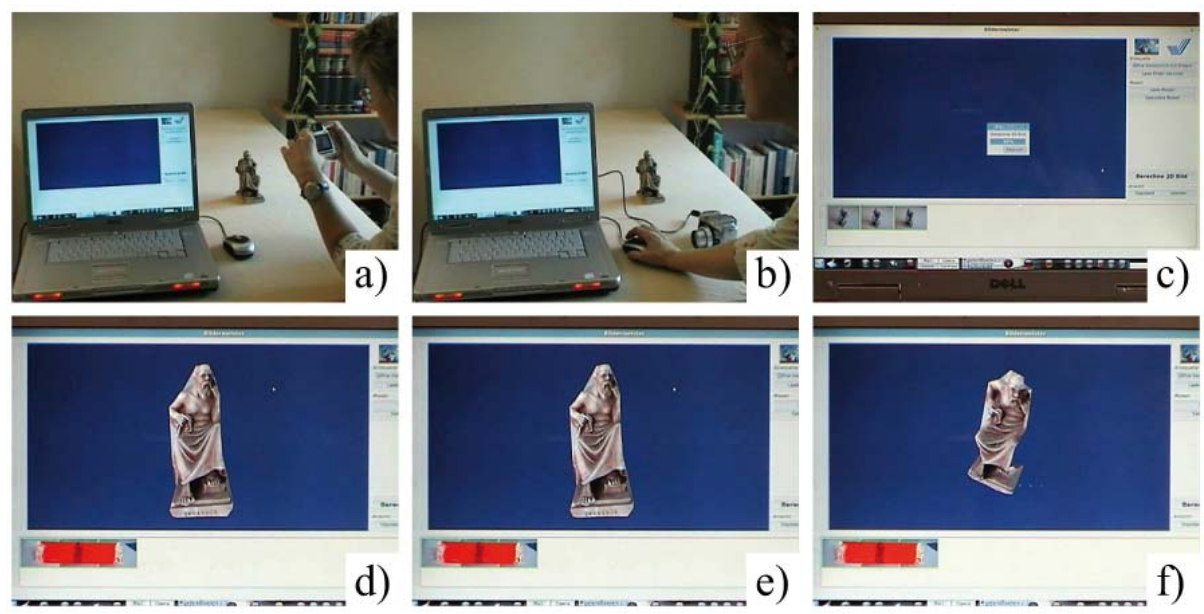

Fig. 2. a) Take snapshots. b) Connect camera to computer, initiate image loading and model creation. c) Progress bar while the model is generated. d) Generated $3 \mathrm{~d}$ model is displayed. e),f) Visualization of model from different viewpoints.

Fig. 2 shows the operation of our system in practice. We hope this tool is not only useful for professional applications but also attractive to the broad public.

\section{References}

1. Saxena, A., Sun, M., Ng, A.Y.: Make3d: Depth perception from a single still image. In: Fox, D., Gomes, C.P. (eds.) AAAI, pp. 1571-1576 (2008)

2. Lowe, D.G.: Distinctive image features from scale-invariant keypoints. Int. J. Comput. Vision 60(2), 91-110 (2004)

3. Fischler, M.A., Bolles, R.C.: Random sample consensus: a paradigm for model fitting with applications to image analysis and automated cartography. Commun. ACM 24(6), 381-395 (1981) 